\section{MicroRNAs: Marcadores séricos en diabetes mellitus tipo 2 y ejercicio físico}

\author{
NICOLÁS GÓMEZ-BANOY ${ }^{1, \mathrm{a}}$, ISMENA MOCKUS ${ }^{1, \mathrm{~b}}$
}

\section{MicroRNAs: circulating biomarkers in type 2 Diabetes Mellitus and physical exercise}

MicroRNAs are small, non-coding molecules with a crucial function in the cell's biologic regulation. Circulating levels of miRNAs may be useful biomarkers in metabolic diseases such as type 2 Diabetes Mellitus (DM2), which alters the circulating concentrations of several types of miRNA. Specific serum profiles of these molecules have been identified in high-risk patients before the development of DM2 and its chronic complications. Most importantly, these profiles can be modified with physical exercise, which is crucial in the treatment of metabolic diseases. Acute physical activity alone can induce changes in tissue specific miRNAs, and responses are different in aerobic or non-aerobic training. Muscle and cardiovascular miRNAs, which may play an important role in the adaptation to exercise, are predominantly altered. Even further, there is a correlation between serum levels of miRNAs and fitness, suggesting a role for chronic exercise in their regulation. Thus, miRNAs are molecules of growing importance in exercise physiology, and may be involved in the mechanisms behind the beneficial effects of physical activity for patients with metabolic diseases.

(Rev Med Chile 2016; 144: 355-363)

Key words: Diabetes Mellitus, Type 2; Exercise; MicroRNAs.

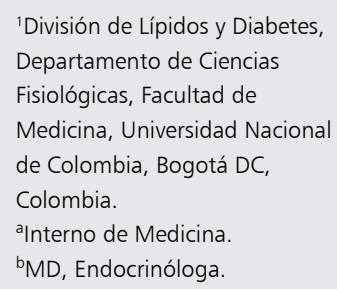

Recibido el 30 de julio de 2015 aceptado el 27 de octubre de 2015

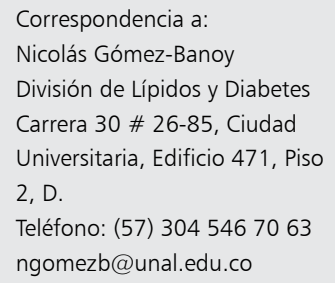

\section{I} a diabetes mellitus tipo 2 (DM2) es una enfermedad metabólica de alta prevalencia en el mundo, y sus complicaciones crónicas conllevan una alta morbi-mortalidad en la población. Para el año 2030, se estima que 552 millones de personas tendrán DM2 en el mundo ${ }^{1}$. En Latinoamérica la prevalencia de la DM2 va en aumento, ligada al incremento de condiciones asociadas como la obesidad y el síndrome metabólico ${ }^{2}$. La fisiopatología de la DM2 es multifactorial; factores genéticos y factores ambientales como el sedentarismo, la obesidad y la nutrición hipercalórica son determinantes en su desarrollo. Los estados pro-inflamatorios, sistémicos, de bajo grado son característicos de las enfermedades metabólicas, incluyendo la $\mathrm{DM}^{3}$. Se cree que el desbalance entre citoquinas pro y anti-inflamatorias puede ser clave en su patogénesis; por esto, varias de estas moléculas han sido propuestas como marcadores tempranos de la enfermedad y sus complicaciones ${ }^{4}$. Sin embargo, muchos de los biomarcadores estudiados carecen de suficiente especificidad, por lo cual no se utilizan de manera masiva ${ }^{5}$.

A pesar de numerosos estudios, todavía no es clara en su totalidad la fisiopatología de la DM2, y no hay tratamiento que la cure. El ejercicio físico se ha destacado como una de las primeras líneas de tratamiento para las enfermedades metabólicas ${ }^{6}, y$ entre los mecanismos para explicar sus beneficios están sus efectos anti-inflamatorios ${ }^{7}$. No obstante, la conexión desde el punto de vista fisiológico y terapéutico entre ejercicio físico y DM2 aún no es clara, especialmente en pre-diabéticos o individuos con factores de riesgo. Por esto, la búsqueda de biomarcadores séricos de DM2 modificables por el ejercicio físico es importante. El objetivo de esta revisión es tratar el tema de los microRNAs (miRNAs) como potenciales biomarcadores en 
el desarrollo de la DM2. Además se quiere dar a conocer el impacto que tiene el ejercicio físico en estas moléculas, tanto en el contexto de esta enfermedad, como en las personas sanas.

\section{MicroRNAs}

Los miRNAs son ácidos ribonucleicos (RNA) no codificantes, pequeños, generalmente compuestos por 21 a 25 nucleótidos. Estudios recientes han encontrado que son parte fundamental de la maquinaria reguladora de la célula y juegan un papel crucial en la mayoría de funciones biológicas $^{8-10}$.

Los miRNAs provienen de secuencias intergénicas del genoma o de intrones en genes codificantes $^{11}$. Inicialmente por acción de la RNA-polimerasa II se forman transcritos primarios de RNA (pri-miRNAs) que son clivados por la ribonucleasa III (Drosha) para dar lugar a estructuras tipo horquilla de alrededor de 70 nucleótidos, los precursores de miRNA (pre-miRNAs) ${ }^{12}$. La exportina 5 media el transporte de los pre-miRNAs del núcleo al citoplasma, donde son clivados por la endoribonucleasa (Dicer) para generar dupletas de aproximadamente 22 nucleótidos consistentes en 1 hebra guía y 1 hebra pasajera ${ }^{13}$. La hebra guía es incorporada al complejo de silenciamiento inducido por RNA (RISC), generando los miRNAs maduros que ejercen sus funciones biológicas, en su mayoría consistentes en inhibición de la traduc- ción de determinados RNA mensajeros (mRNA). La hebra pasajera es degradada en la mayoría de los $\operatorname{casos}^{9}$ (Figura 1).

El miRNA maduro asociado al RISC se une mediante reconocimiento de secuencias complementarias a nivel de la región $3 `$ no transcrita del mRNA objetivo. Estas secuencias se han denominado "secuencias semillas" y son residuos de 2-8 nucleótidos. El resultado puede ser la degradación del mRNA objetivo o la inhibición de su traducción, dependiendo de la complementariedad de las secuencias ${ }^{14}$ (Figura 1).

Los miRNA pueden encontrarse en el suero humano y pueden ser biomarcadores debido a su alta homogeneidad y estabilidad ${ }^{15,16}$. Inicialmente se identificó que los mecanismos de transporte y liberación de estas moléculas se dan mediante la formación y liberación de exoxomas que se producen por una vía dependiente de ceramidas ${ }^{17}$. Posteriormente se propuso que los miRNAs son transportados en el plasma por proteínas de unión específicas ${ }^{18} \mathrm{o}$ lipoproteínas como la lipoproteína de alta densidad (HDL) ${ }^{19}$.

\section{MicroRNAs como biomarcadores séricos en DM2}

Los miRNAs han sido utilizados como biomarcadores en patologías como el cáncer y enfermedades auto-inmunes ${ }^{20,21}$. Los primeros trabajos para establecer un patrón de los miRNA circulantes en

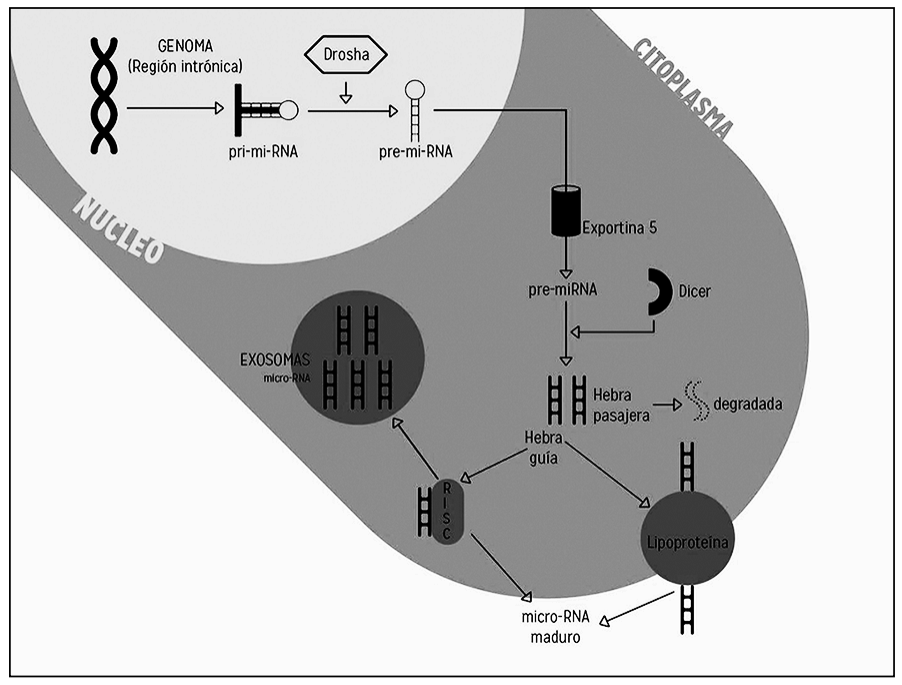

Figura 1. Síntesis de micro-RNA. Los miRNAs provienen de secuencias intergénicas del genoma 0 de intrones en genes codificantes ${ }^{11}$. Los transcritos primarios de RNA (pri-miRNAs) son clivados por la ribonucleasa III (Drosha) para dar lugar a estructuras de alrededor de 70 nucleótidos, los precursores de miRNA (pre-miRNAs) ${ }^{12}$. La exportina 5 media el transporte de los pre-miRNAs del núcleo al citoplasma, donde son clivados por la endoribonucleasa (Dicer) para generar dupletas de aproximadamente 22 nucleótidos consistentes en 1 hebra guía y 1 hebra pasajera ${ }^{13}$. La hebra guía es incorporada al complejo de silenciamiento inducido por RNA (RISC), generando los miRNAs maduros que ejercen sus funciones biológicas. La hebra pasajera es degradada en la mayoría de los $\operatorname{casos}^{9}$. Los miRNAs maduros también se pueden acoplar a lipoproteínas o exoxomas para su transporte fuera de la célula17,19. 
DM2 fueron efectuados por Zampetaki y cols., quienes identificaron 13 miRNAs candidatos en una población de 822 sujetos; posteriormente los cuantificaron en el suero de pacientes con DM2 $(\mathrm{n}=80)$ y controles sanos $(\mathrm{n}=80)$. Encontraron que 11 miRNAs presentaban cambios significativos en DM2, sin embargo, cinco (miR-15a, miR28-3p, miR-29b, miR-126 y miR-223) presentaban la suficiente validez estadística para ser utilizados como biomarcadores en individuos con DM2 diagnosticada. De manera interesante, estos 5 miRNAs se encontraban desregulados en 19 pacientes que desarrollaron diabetes a lo largo del estudio, y también se postularon como biomarcadores tempranos de DM222. Específicamente, el miR-126 ha sido identificado como regulador de procesos angiogénicos en los vasos sanguíneos periféricos ${ }^{23,24}$. Zampetaki y cols., midieron esta molécula en toda su población de estudio $(\mathrm{n}=822)$, observando niveles significativamente menores en los diabéticos.

Más adelante, Kong y cols., analizaron $7 \mathrm{miR}-$ NAs asociados a DM2 (miR-9, miR-29a, miR-30d, miR-34a, miR-124a, miR-146a y miR-375) en sujetos con DM2 recién diagnosticada $(\mathrm{n}=18)$, pre-diabéticos $(\mathrm{n}=19)$ y normoglucémicos con factores de riesgo para desarrollar DM2 $(n=19)$. Confirmaron que los 7 miRNA estudiados presentaban niveles circulantes significativamente mayores en los DM2 comparados con los pre-diabéticos y normoglucémicos. Sin embargo, no encontraron diferencia entre pre-diabéticos y normoglucémicos, y concluyeron que las diferencias en el perfil de miRNAs circulantes entre estos dos estados no eran significativas ${ }^{25}$.

Los miRNAs también han sido cuantificados en pacientes con síndrome metabólico. Karolina y cols., analizaron la concentración sanguínea de 200 miRNAs en una cohorte de pacientes $(\mathrm{n}=265)$ con síndrome metabólico $(\mathrm{n}=50)$, DM2 $(\mathrm{n}=50)$, hipercolesterolemia $(\mathrm{n}=89)$, hipertensión $(n=30)$, y controles sanos $(n=46)$. En el grupo de DM2, cinco miRNAs se encontraron aumentados (miR-150, miR-192, miR-27a, miR-320a, y miR-375). De manera interesante, encontraron que en algunos pacientes con síndrome metabólico y una glucemia alterada (sin DM2), los niveles de glucemia basal elevados se correlacionaban positivamente con dos miRNA: miR-27a y miR-320a ${ }^{26}$. En la misma línea, Pescador y cols., determinaron el perfil de miRNAs circulante en una cohorte de 69 sujetos con DM2 $(n=13)$, obesos $(n=20)$, obesos con DM2 $(\mathrm{n}=16)$ y controles sanos $(\mathrm{n}=20)$. Encontraron que la combinación de miR-503 y miR-138 podía ser utilizada para distinguir pacientes con DM2 y obesos, de pacientes con DM2 sin obesidad ${ }^{27}$. Los hallazgos de los dos estudios anteriores abren la puerta a la utilización de miRNAs como marcadores que permiten distinguir enfermedades fuertemente relacionadas, como son DM2, obesidad y síndrome metabólico. A su vez, Zhang y cols., corroboraron en un estudio de 90 sujetos (30 DM2, 30 pre-diabéticos y 30 controles) que el miR-126 se encontraba significativamente disminuido en los pacientes pre-diabéticos y diabéticos con respecto a los controles. De esta manera el miR-126 es propuesto como factor de riesgo independiente para desarrollar DM2 en individuos susceptibles, y como posible biomarcador temprano de DM2 ${ }^{28}$.

Los estudios de miRNAs como marcadores de DM2 se han realizado en otros grupos poblacionales. Recientemente, Wang y cols., compararon las concentraciones de 14 miRNAs en sujetos suecos e iraquís con DM2, pre-diabetes y normoglucémicos. Un total de 152 personas fueron reclutadas, diecinueve de los 84 iraquís y catorce de los 68 suecos estudiados presentaban DM2. De las moléculas estudiadas, sólo los miR-24 y miR-29-b se asociaban a DM2 en el total de la población. De manera interesante, las concentraciones séricas elevadas de miR-144 se asociaban a DM2 únicamente en los pacientes suecos, sugiriendo que su asociación con DM2 es específica de esta etnia ${ }^{29}$.

Esto, según los autores, abre la puerta a una posible expresión específica de determinados miRNAs en las distintas etnias.

Recientemente, se han publicado 2 estudios que analizaron los cambios de los miRNAs en respuesta al tratamiento de la DM2. En el primero, Liu y cols., evaluaron la concentración sérica del miRNA-126 en pacientes con pre-diabetes $(\mathrm{n}=157)$, DM2 recientemente diagnosticada $(\mathrm{n}=160)$ y sanos $(\mathrm{n}=138)$. Identificaron que el miR-126 se encontraba significativamente disminuido en los pacientes con DM2 y pre-diabetes. Sin embargo, de manera muy interesante, después de realizar intervenciones terapéuticas durante 6 meses (dieta y ejercicio en los pre-diabéticos, terapia insulínica más dieta y ejercicio en los DM2), los niveles de miR-126 aumentaron significativamente en ambos grupos, especialmente en los DM2 ${ }^{30}$. 
El segundo estudio fue realizado por Párrizas y cols., quienes analizaron 176 miRNAs en una población de DM2 $(\mathrm{n}=10)$, pre-diabéticos $(\mathrm{n}=19)$ divididos en aquellos con hiperglucemia en ayunas (IFG) $(\mathrm{n}=10)$ e intolerancia a los carbohidratos (IGT) $(n=9)$, y sujetos control $(n=17)$. Como hallazgo innovador, encontraron 3 miRNAs cuyas concentraciones séricas estaban significativamente elevadas exclusivamente en los pre-diabéticos (IFG e IGT); miR-150, miR-192 y miR-193b. Análisis estadísticos posteriores revelaron que los dos últimos presentaban mayor significancia estadística, postulándolos como posibles biomarcadores para distinguir el fenotipo de pre-diabetes. De manera muy interesante, 6 pre-diabéticos y 13 controles participaron en un protocolo de ejercicio supervisado y recomendaciones dietarias por 16 semanas. Al final de la intervención, los sujetos pre-diabéticos presentaban una disminución significativa de sus niveles séricos basales de miR-192 y miR-193b, llegando a ser comparables con los controles $^{31}$. Ambos estudios, además de proponer un grupo de miRNAs (miRNA-126, miRNA-192, miRNA-193b) que son potenciales marcadores tempranos de DM2, demuestran un posible efecto del ejercicio físico sobre estas moléculas. Como ya se mencionó, el miRNA-126 es un importante regulador a nivel del sistema cardiovascular ${ }^{23,24}$. Por otro lado, el miRNA-192 es una molécula predominantemente hepática ${ }^{32}$, y puede indicar disfunción en patologías como la esteatosis hepática $^{33}$. En cambio, el miRNA-193b es una molécula propia del tejido adiposo, y es importante en su diferenciación ${ }^{34}$ (Figura 2).

\section{MicroRNAs circulantes y ejercicio físico}

El ejercicio físico es considerado una de las primeras opciones de tratamiento en $\mathrm{DM} 2^{6}$ por sus propiedades anti-inflamatorias ${ }^{7}$. Estudios recientes sobre la fisiología de los miRNAs en las enfermedades metabólicas ha abierto la puerta a un posible rol de estas moléculas en el ejercicio físico y sus efectos terapéuticos. Se han determinado concentraciones basales de miRNAs en diferentes poblaciones, así como su respuesta a ejercicio físico aeróbico y anaeróbico.

\section{Mecanismos de liberación de microRNAs circulantes durante ejercicio físico}

Uno de los principales dilemas al analizar los trabajos de miRNAs y ejercicio físico es el origen de estas moléculas circulantes. Todavía no es claro si sus cambios con la actividad física se deben a daño

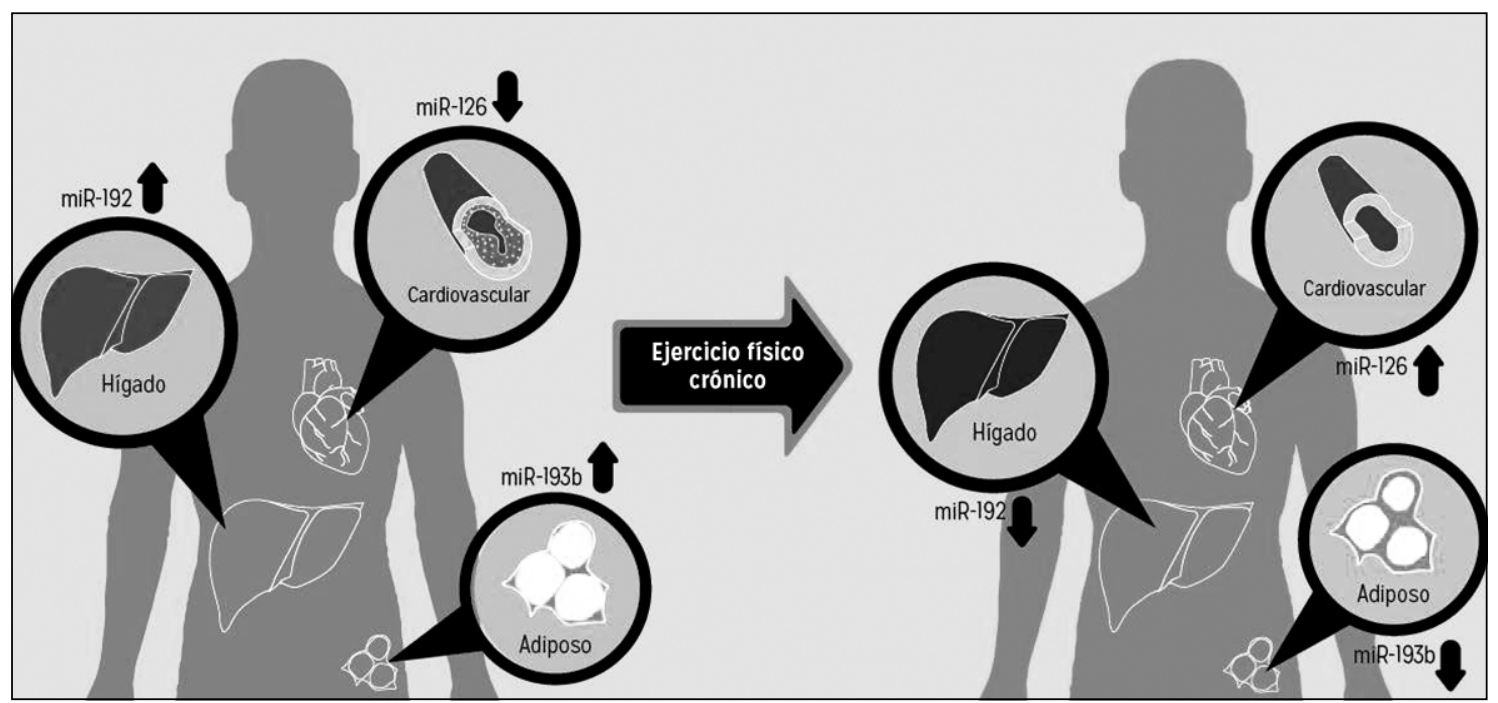

Figura 2. Pre-diabetes, micro-RNAs y ejercicio físico. Los sujetos pre-diabéticos sedentarios presentan niveles elevados de miR192, asociado a la esteatosis hepática ${ }^{33}$, y miR-193b molécula específica de tejido adiposo ${ }^{34}$. Además presentan disminución de miR-126, molécula reguladora de procesos angiogénicos ${ }^{23,24}$. La realización de ejercicio físico de $4^{30}$ a 6 meses ${ }^{31}$ puede llevar a cambios en el perfil de estos miRNAs, disminuyendo los niveles de miR-192 y miR-193b, y aumentado los niveles de miR-126. Los cambios en estas moléculas pueden estar asociadas a los efectos benéficos del ejercicio físico en estos órganos. 
celular y liberación de miRNAs intracelulares, o a secreción activa de las mismas por parte de tejidos específicos como respuesta fisiológica al ejercicio.

Inicialmente, Baggish y cols., cuantificaron la respuesta de miRNAs circulantes al ejercicio físico agudo exhaustivo (realizado en bicicleta estática) en un grupo de remeros $(\mathrm{n}=10)$ antes y después de 90 días de entrenamiento. Identificaron miRNAs que aumentaban sus concentraciones circulantes con el ejercicio físico agudo (miR-146 y miR-222), y con la actividad física aguda exclusivamente antes del período de entrenamiento (miR-21 y miR-221). Otros respondían exclusivamente al ejercicio crónico (miR-20a). Debido a esta expresión diferencial de miRNAs entre ejercicio agudo y crónico, los autores concluyen que el mecanismo de liberación de miRNAs séricos debía ser más complejo que la simple liberación por daño celular ${ }^{35}$.

Los estudios con ejercicio físico anaeróbico sugieren que los miRNAs circulantes son producto de destrucción celular. Ulheman encontró que el ejercicio de resistencia muscular anaeróbico (cargas excéntricas) generaba incremento de miR-133 (específico de músculo esquelético) ${ }^{36}$. Banzet y cols., también identificaron elevación de miRNAs específicos de músculo esquelético (hsa-miR-1, miR-133a, miR-133b y miR-208b) con ejercicio muscular excéntrico (caminata cuesta abajo), el cual se asocia a daño muscular ${ }^{37}$. En contraste, Sawada y cols., no reportaron incremento de los mio-miRNAs en respuesta a un ejercicio de resistencia muscular anaeróbico (levantamiento de pesas con piernas) ${ }^{38}$ (Tabla 1$)$.

Por el contrario, investigaciones realizadas con ejercicio físico aeróbico sugieren mecanismos de liberación más complejos. Nielsen y cols., midieron 188 miRNAs en respuesta a ejercicio aeróbico en sujetos daneses sanos. Los resultados sugieren una secreción selectiva de miRNAs al torrente circulatorio, pues se reportaron elevaciones de algunas moléculas específicas de músculo, además de moléculas específicas de hígado ${ }^{39}$. Baggish reportó hallazgos similares en sujetos entrenados que participaron en una maratón. Encontraron elevaciones de algunos miRNAs específicos de músculo esquelético, miocardio y procesos inflamatorios, sin presentarse cambios en otros miRNAs de los mismos tejidos (Tabla 2$)^{40}$. Además compararon los miRNAs que sufrían cambios con marcadores convencionales de daño tisular: creatina fosfoquinasa (daño muscular), troponina $\mathrm{T}$ (daño miocárdico), pro-péptido natriurético $\mathrm{B}$ (estrés tejido miocárdico) y proteína $\mathrm{C}$ reactiva (marcador general de inflamación). Encontraron que el comportamiento biológico de los miRNAs era muy distinto al de los marcadores habituales, pues si bien ambos grupos de moléculas incrementaron de manera significativa sus niveles con el ejercicio, los miRNAs aumentaron inmediatamente, y disminuyeron sus valores $24 \mathrm{~h}$ posterior al ejercicio, mientras que los marcadores habituales se elevaron más lentamente y permanecieron elevados $24 \mathrm{~h}$ después de terminado el ejercicio. Lo anterior fortalece la idea de que los miRNAs son marcadores en tiempo real de ejercicio físico, con potencial rol biológico, más que simples marcadores de daño tisular ${ }^{40}$.

Si bien hay pocos estudios, se puede vislumbrar un patrón de liberación de los miRNAs dependiendo del tipo de ejercicio físico. Los miRNAs específicos de tejidos, especialmente de músculo esquelético, se elevan de manera importante durante la realización de ejercicio físico anaeróbico, el cual se asocia a daño muscular. Posiblemente sea en este tipo de ejercicio físico que la liberación pasiva de miRNAs por muerte celular predomine. Por otro lado, en el ejercicio físico aeróbico se

Tabla 1. Respuesta de microRNAs a ejercicio físico anaeróbico agudo

\begin{tabular}{|c|c|c|c|c|c|}
\hline Estudio & $\begin{array}{l}\text { miRNAs de músculo } \\
\text { esquelético }\end{array}$ & $\begin{array}{l}\text { miRNAs de } \\
\text { inflamación }\end{array}$ & $\begin{array}{l}\text { miRNAs de } \\
\text { miocardio }\end{array}$ & $\begin{array}{l}\text { miRNAs } \\
\text { hepáticos }\end{array}$ & $\begin{array}{l}\text { miRNAs de angio- } \\
\text { génesis/endotelio }\end{array}$ \\
\hline Uhlemann ${ }^{28}$ & $\uparrow$ miR-133 en T0 & & & & \\
\hline Banzet $^{29}$ & $\begin{array}{l}\uparrow \text { hsa-miR-1, miR-133a, } \\
\text { miR-133b y miR-208B } \\
\text { 2-6 HD }\end{array}$ & & & & \\
\hline Sawada ${ }^{30}$ & & $\downarrow$ miR-146a, tres DD & & & $\downarrow$ miR-221, tres DD \\
\hline
\end{tabular}

Siglas: TO: Tiempo 0; HD: Horas después; DD: Días después. $\uparrow$ : elevación significativa. $\downarrow$ : disminución significativa 
Tabla 2. Respuesta de miRNAs a ejercicio físico agudo aeróbico

\begin{tabular}{|c|c|c|c|c|c|}
\hline Estudio & $\begin{array}{l}\text { miRNAs de } \\
\text { músculo } \\
\text { esquelético }\end{array}$ & $\begin{array}{l}\text { miRNAs de } \\
\text { inflamación }\end{array}$ & $\begin{array}{l}\text { miRNAs de } \\
\text { miocardio }\end{array}$ & $\begin{array}{l}\text { miRNAs } \\
\text { hepáticos }\end{array}$ & $\begin{array}{l}\text { miRNAs de } \\
\text { angiogénesis/ } \\
\text { endotelio }\end{array}$ \\
\hline Baggish $^{27}$ & $\operatorname{miR}-133 \leftrightarrow$ & $\begin{array}{l}\uparrow \operatorname{miR}-21 \\
A E \\
\uparrow \operatorname{miR}-146 a \\
A E \text { y } D E\end{array}$ & & & $\begin{array}{l}\uparrow \operatorname{miR}-222 \\
A E \text { y } D E \\
\uparrow \text { miR-221 } \\
A E\end{array}$ \\
\hline Uhleman ${ }^{28}$ & & & & & $\uparrow \operatorname{miR}-126$ \\
\hline Nielsen ${ }^{31}$ & $\begin{array}{l}\uparrow \text { miR-1, } \\
\text { miR-133a, } \\
\text { miR-133 } \\
\text { Tres HD }\end{array}$ & $\uparrow \operatorname{miR}-146 a$ & $\begin{array}{l}\uparrow \text { miR-223 } \\
\text { Una HD }\end{array}$ & $\begin{array}{l}\downarrow \text { miR-151-5p } \\
\text { en T0 } \\
\uparrow \text { miR-145, } \\
\text { miR-143 } \\
\text { Una HD }\end{array}$ & $\downarrow$ miR-221 \\
\hline Baggish $^{3}$ & $\begin{array}{l}\uparrow \text { miR-1, } \\
\text { miR-133 en T0 } \\
\text { miR-499-5p } \leftrightarrow\end{array}$ & $\uparrow \operatorname{miR}-146$ & $\uparrow \operatorname{miR}-208$ & & $\operatorname{miR}-126 \leftrightarrow$ \\
\hline Mooren 33 & $\begin{array}{l}\uparrow \text { miR-1, miR-133, } \\
\text { miR-206 en T0 }\end{array}$ & $\begin{array}{l}\operatorname{miR}-21 \\
\operatorname{miR}-155 \leftrightarrow\end{array}$ & $\begin{array}{l}\uparrow \text { miR-208b, } \\
\text { miR-499 en T0 }\end{array}$ & & \\
\hline
\end{tabular}

Siglas: AE: antes de entrenamiento; DE: después de entrenamiento; HD: horas después; TO: tiempo 0 . $\uparrow:$ elevación significativa. $\downarrow$ : disminución significativa. $\leftrightarrow$ : no presentó cambios.

evidencia un reclutamiento de variedad de tejidos y aumento en las concentraciones de varios tipos de miRNAs. Se podría sugerir que estas moléculas son secretadas selectiva y activamente por los tejidos, y podrían jugar un papel fundamental en la regulación fisiológica del ejercicio físico aeróbico.

\section{Fisiología de microRNAs circulantes con el ejercicio físico}

En diferentes estudios se han identificado diferentes patrones de miRNAs circulantes dependiendo del tipo de ejercicio físico y del tipo de personas que lo realizan.

El análisis del ejercicio aeróbico y los patrones de miRNAs circulantes ha mostrado resultados interesantes. Baggish reportó elevación de miR-146y miR-221 en respuesta a ejercicio aeróbico exhaustivo en bicicleta, con incremento progresivo de la intensidad $^{35}$. En un segundo estudio, se observó incremento del miR-146 en respuesta a una maratón ${ }^{40}$. Por otro lado, Nielsen y cols., reportaron disminución de los mismos miRNAs posterior a ejercicio en bicicleta estática a $65 \%$ del consumo máximo de oxígeno (VO2max) durante $1 \mathrm{~h}^{39}$. El miR-146 es una molécula reguladora del sistema inmune innato, y entre sus proteínas blanco se encuentra al factor 6 asociado al receptor del factor de necrosis tumoral (TRAF6) y las quinasas 1 y 2 asociadas al receptor de interleuquina 1 (IRAK 1 y 2$)^{41}$, mientras que el miR-221 es importante en la regulación de angiogénesis ${ }^{42}$. Las diferencias de los resultados entre los estudios mencionados podría ser explicada por la intensidad de ejercicio físico realizado, mucho mayor en los estudios de Baggish. Por otro lado, el miR-126 ha presentado resultados contradictorios. Ulheman y cols., reportaron su elevación ${ }^{36}$ en ejercicio físico aeróbico a distintas intensidades, mientras que en su segundo estudio Baggish no observó cambios de esta molécula ${ }^{40}$. Es evidente que el ejercicio físico aeróbico induce cambios en miRNAs de inflamación y angiogénesis, y esto está directamente implicado en la adaptación fisiológica del organismo a la actividad física (Tabla 2).

Como se mencionó antes, llama la atención la elevación de algunos marcadores de músculo esquelético con el ejercicio aeróbico. Nielsen ${ }^{39}$ reportó elevación importante, aunque no significativa, de mio-miRNAs en los tiempos 0 , y tres horas después del ejercicio. Baggish ${ }^{40}$ y Mooren ${ }^{43}$ reportaron elevación de miRNAs de músculo esquelético. Es muy interesante observar que en estos 3 estudios, la maratón fue el ejercicio realizado, caracterizado por alta intensidad, duración 
prolongada, y máxima exigencia cardiovascular y muscular ${ }^{39,40,43}$. Así, se podría concluir que el ejercicio aeróbico genera cambios en la expresión de miRNAs asociados al músculo, predominantemente cuando la intensidad del mismo es máxima y se produce cierto grado de daño muscular. El papel biológico de miR-1 y miR-133 está probablemente asociado a la remodelación del este tejido ${ }^{44}$.

En cuanto al ejercicio físico anaeróbico, los estudios muestran los resultados ya expuestos (Tabla 1), con predominio en la elevación de miRNAs específicos de músculo esquelético ${ }^{35,36}$.

Una de las maneras de cuantificar el estado físico de un sujeto es mediante el VO2max. Son de gran importancia los estudios que asocian este parámetro corporal con los miRNAs. Bye y cols., determinaron que el miR-210 (hipoxia), miR-21 (inflamación), y miR-222 (angiogénesis), se encontraban aumentados en los sujetos con VO2max baja ${ }^{45}$. De esta manera, estas moléculas fueron postuladas como marcadores tempranos de enfermedad cardiovascular o sedentarismo. Muy recientemente, Wardle y cols., compararon niveles basales de miRNAs en individuos sedentarios, atletas de fondo y atletas de fuerza. Observaron que los atletas de fondo presentaban niveles mayores de miR-222 y miR-221 (angiogénesis) así como miR-21 y miR-146 ${ }^{\text {a }}$ inflamación) ${ }^{46}$. Los dos estudios demuestran cómo se presentan cambios de moléculas de inflamación y angiogénesis con el ejercicio aeróbico crónico, similar al ejercicio físico agudo.

En conclusión, los cambios en la expresión de un grupo de miRNAs varían según el ejercicio es aeróbico o anaeróbico y se pueden correlacionar con la fisiología del ejercicio. Además, los miRNAs séricos provienen de tejidos específicos, donde ejercen acciones importantes para el funcionamiento de los mismos. Es necesaria la realización de estudios a largo plazo sobre la correlación de estas moléculas circulantes con parámetros de salud cardiovascular y metabólica y así establecer su rol en los efectos benéficos del ejercicio físico. Sería interesante involucrar en este tipo de investigaciones a sujetos no atletas, quienes pertenecen a la población que probablemente se beneficiaría más del ejercicio físico para prevenir patologías cardiovasculares y metabólicas. Los hallazgos sobre el efecto del ejercicio físico en personas con trastornos de los carbohidratos ${ }^{30,31}$, hacen que los miRNAs sean moléculas de obligatorio estudio en investigaciones sobre ejercicio físico y sus efectos terapéuticos en las enfermedades metabólicas.

Agradecimientos: Los autores expresan su agradecimiento a la diseñadora Ana María Gómez Correal por su colaboración durante la realización de las figuras del presente artículo.

\section{Referencias}

1. Whiting DR, Guariguata L, Weil C, Shaw J. IDF diabetes atlas: global estimates of the prevalence of diabetes for 2011 and 2030. Diabetes Res Clin Pract 2011; 94 (3): 311-21.

2. Cuevas A, Álvarez V, Carrasco F. Epidemic of metabolic syndrome in Latin America. Curr Opin Endocrinol Diabetes Obes 2011; 18 (2): 134-8.

3. Hotamisligil GS. Inflammation and metabolic disorders. Nature 2006; 444: 860-7.

4. Schulze MB, Weikert C, Pischon T, Bergmann MM, Al-Hasani H, Schleicher E, et al. Use of multiple metabolic and genetic markers to improve the prediction of type 2 diabetes: the EPIC Potsdam Study. Diabetes Care 2009; 32 (11): 2116-9.

5. Kolberg JA, Jørgensen T, Gerwien RW, Hamren S, McKenna MP, Moler E, et al. Development of a type 2 diabetes risk model from a panel of serum biomarkers from the Inter 99 cohort. Diabetes Care 2009; 32 (7): 1207-12.

6. Church T. Exercise in obesity, metabolic syndrome, and diabetes. Prog Cardiovasc Dis 2011; 53 (6): 412-8.

7. Petersen AM, Pedersen BK. The anti-inflammatory effect of exercise. J Appl Physiol 2005; 98 (4): 1154-62.

8. Flynt AS, Lai EC. Biological principles of microRNA-mediated regulation: shared themes amid diversity. Nat Rev Genet 2008; 9 (11): 831-42.

9. Bartel DP. MicroRNAs: genomics, biogenesis, mechanism, and function. Cell 2004; 116 (2): 281-97.

10. Chua JH, Armugam A, Jeyaseelan K. MicroRNAs: biogenesis, function and applications. Curr Opin Mol Ther 2009; 11 (2): 189-99.

11. Rodríguez A, Griffiths-Jones S, Ashurst JL, Bradley A. Identification of mammalian microRNA host genes and transcription units. Genome Res 2004; 14 (10A): 190210.

12. Lee $\mathrm{Y}, \mathrm{Kim} \mathrm{M}, \mathrm{Han} \mathrm{J}$, Yeom KH, Lee S, Baek SH, et al. MicroRNA genes are transcribed by RNA polymerase II. EMBO J 2004; 23 (20): 4051-60.

13. Kim VN, Han J, Siomi MC. Biogenesis of small RNAs in animals. Nat Rev Mol Cell Biol 2009; 10 (2): 126-39. 
14. Doench JG, Sharp PA. Specificity of microRNA target selection in translational repression. Genes Dev 2004; 18 (5): 504-11.

15. Mitchell PS, Parkin RK, Kroh EM, Fritz BR, Wyman SK, Pogosova-Agadjanyan EL, et al. Circulating microRNAs as stable blood-based markers for cancer detection. Proc Natl Acad Sci U S A. 2008; 105 (30): 10513-8.

16. Keller A, Leidinger P, Bauer A, Elsharawy A, Haas J, Backes C, et al. Toward the blood-borne miRNome of human diseases. Nat Methods 2011; 8 (10): 841-3.

17. Kosaka N, Iguchi H, Yoshioka Y, Takeshita F, Matsuki Y, Ochiya T. Secretory mechanisms and intercellular transfer of microRNAs in living cells. J Biol Chem 2010; 285 (23): 17442-52.

18. Arroyo JD, Chevillet JR, Kroh EM, Ruf IK, Pritchard CC, Gibson DF, et al. Argonaute2 complexes carry a population of circulating microRNAs independent of vesicles in human plasma. Proc Natl Acad Sci U S A. 2011; 108 (12): 5003-8.

19. Vickers KC, Palmisano BT, Shoucri BM, Shamburek $\mathrm{RD}$, Remaley AT. MicroRNAs are transported in plasma and delivered to recipient cells by high-density lipoproteins. Nat Cell Biol 2011; 13 (4): 423-33.

20. Wang J, Zhang KY, Liu SM, Sen S. Tumor-associated circulating microRNAs as biomarkers of cancer. Molecules 2014; 19 (2): 1912-38.

21. Qu Z, Li W, Fu B. MicroRNAs in autoimmune diseases. Biomed Res Int 2014; 2014: 527895.

22. Zampetaki A, Kiechl S, Drozdov I, Willeit P, Mayr U, Prokopi M, et al. Plasma microRNA profiling reveals loss of endothelial miR-126 and other microRNAs in type 2 diabetes. Circ Res 2010; 107 (6): 810-7.

23. Wang S, Aurora Richardson JA, Bassel-Duby R, Olson EN. AB, Johnson BA, Qi X, et al. The endothelial-specific microRNA miR-126 governs vascular integrity and angiogenesis. Dev Cell 2008; 15 (2): 261-71.

24. Mocharla P, Briand S, Giannotti G, Dörries C, Jakob P, Paneni F, et al. AngiomiR-126 expression and secretion from circulating CD34(+) and CD14(+) PBMCs: role for proangiogenic effects and alterations in type 2 diabetics. Blood 2013; 121 (1): 226-36.

25. Kong L, Zhu J, Han W, Jiang X, Xu M, Zhao Y, et al. Significance of serum microRNAs in pre-diabetes and newly diagnosed type 2 diabetes: a clinical study. Acta Diabetol 2011; 48 (1): 61-9.

26. Karolina DS, Tavintharan S, Armugam A, Sepramaniam S, Pek SL, Wong MT, et al. Circulating miRNA profiles in patients with metabolic syndrome. J Clin Endocrinol Metab 2012; 97 (12): E2271-6.

27. Pescador N, Pérez-Barba M, Ibarra JM, Corbatón A, Martínez-Larrad MT, Serrano-Ríos M. Serum circula- ting microRNA profiling for identification of potential type 2 diabetes and obesity biomarkers. PLoS One 2013; 8 (10): e77251.

28. Zhang T, Lv C, Li L, Chen S, Liu S, Wang C, et al. Plasma miR-126 is a potential biomarker for early prediction of type 2 diabetes mellitus in susceptible individuals. Biomed Res Int 2013; 2013: 761617.

29. Wang X, Sundquist J, Zöller B, Memon AA, Palmér K, Sundquist K, et al. Determination of 14 circulating microRNAs in Swedes and Iraqis with and without diabetes mellitus type 2. PLoS One 2014; 9 (1): e86792.

30. Liu Y, Gao G, Yang C, Zhou K, Shen B, Liang H, et al. The role of circulating microRNA-126 (miR-126): a novel biomarker for screening prediabetes and newly diagnosed type 2 diabetes mellitus. Int J Mol Sci 2014; 15 (6): 10567-77.

31. Párrizas M, Brugnara L, Esteban Y, González-Franquesa A, Canivell S, Murillo S, et al. Circulating miR-192 and miR-193b Are Markers of Prediabetes and Are Modulated by an Exercise Intervention. J Clin Endocrinol Metab 2015; 100 (3): E407-15.

32. van de Bunt M, Gaulton KJ, Parts L, Moran I, Johnson PR, Lindgren CM, et al. The miRNA profile of human pancreatic islets and beta-cells and relationship to type 2 diabetes pathogenesis. PLoS One 2013; 8 (1): e55272.

33. Pirola CJ, Fernández Gianotti T, Castaño GO, Mallardi P, San Martino J, Mora González López Ledesma M, et al. Circulating microRNA signature in non-alcoholic fatty liver disease: from serum non-coding RNAs to liver histology and disease pathogenesis. Gut 64 (5): 800-12.

34. Sun L, Xie H, Mori MA, Alexander R, Yuan B, Hattangadi SM, et al. MiR193b-365 is essential for brown fat differentiation. Nat Cell Biol 2011; 13 (8): 958-65.

35. Baggish AL, Hale A, Weiner RB, Lewis GD, Systrom D, Wang F, et al. Dynamic regulation of circulating microRNA during acute exhaustive exercise and sustained aerobic exercise training. J Physiol 201; 589 (Pt 16): 3983-94.

36. Uhlemann M, Möbius-Winkler S, Fikenzer S, Adam J, Redlich M, Möhlenkamp S, et al. Circulating microRNA-126 increases after different forms of endurance exercise in healthy adults. Eur J Prev Cardiol 2014; 21 (4): 484-91.

37. Banzet S, Chennaoui M, Girard O, Racinais S, Drogou $\mathrm{C}$, Chalabi $\mathrm{H}$, et al. Changes in circulating microRNAs levels with exercise modality. J Appl Physiol (1985) 2013; 115 (9): 1237-44.

38. Sawada S, Kon M, Wada S, Ushida T, Suzuki K, Akimoto T. Profiling of circulating microRNAs after a bout of 
acute resistance exercise in humans. PLoS One 2013; 8 (7): e70823.

39. Nielsen S, Åkerström T, Rinnov A, Yfanti C, Scheele C, Pedersen BK, et al. The miRNA plasma signature in response to acute aerobic exercise and endurance training. PLoS One 2014; 9 (2): e87308.

40. Baggish AL, Park J, Min PK, Isaacs S, Parker BA, Thompson PD, et al. Rapid upregulation and clearance of distinct circulating microRNAs after prolonged aerobic exercise. J Appl Physiol (1985) 2014; 116 (5): 522-31.

41. Labbaye C, Testa U. The emerging role of miR-146A in the control of hematopoiesis, immune function and cancer. J Hematol Oncol 2012; 5: 13.

42. Poliseno L, Tuccoli A, Mariani L, Evangelista M, Citti L, Woods K, et al. MicroRNAs modulate the angiogenic properties of HUVECs. Blood 2006; 108 (9): 3068-71.

43. Mooren FC, Viereck J, Krüger K, Thum T. Circulating microRNAs as potential biomarkers of aerobic exercise capacity. Am J Physiol Heart Circ Physiol 2014; 306 (4): H557-63.

44. Wang XH. MicroRNA in myogenesis and muscle atrophy. Curr Opin Clin Nutr Metab Care 2013; 16 (3): 258-66.

45. Bye A, Røsjø H, Aspenes ST, Condorelli G, Omland T, Wisløff U. Circulating microRNAs and aerobic fitness-the HUNT-Study. PLoS One 2013; 8 (2): e57496.

46. Wardle SL, Bailey ME, Kilikevicius A, Malkova D, Wilson RH, Venckunas T, et al. Plasma MicroRNA Levels Differ between Endurance and Strength Athletes. PLoS One 2015; 10 (4): e0122107. 